\title{
28. Angular Cluster Sets and Horocyclic Angular Cluster Sets
}

\author{
By Hidenobu YoshidA \\ Department of Mathematics, Chiba University, Chiba \\ (Comm. by Kinjirô KUnUGI, M. J. A., Jan. 12, 1971)
}

1. In [1] Bagemihl began a study of relations between nontangential (angular) boundary behaviors and horocyclic boundary behaviors of meromorphic functions defined in the open unit disk $D$ of the complex plane. This study has been continued by Dragosh in [2] and [3]. The purpose of the present paper is to sharpen some of results of these investigations by the method of Dolzhenko's paper.

Notation and definitions. Unless otherwise stated, $f: D \rightarrow W$ shall mean $f(z)$ is an arbitrary function (generally not unique) defined in the open unit disk $D:|z|<1$ and assuming values in the extended complex plane $W$. The unit circle $|z|=1$ is denoted by $\Gamma$.

A circle internally tangent to $\Gamma$ at a point $\zeta \in \Gamma$ is called a horocycle at $\zeta$, and will be denoted by $h_{r}(\zeta)$, where $r(0<r<1)$ is the radius of the horocycle.

Given a horocycle $h_{r}(\zeta)$ at a point $\zeta \in \Gamma$, the region interior to $h_{r}(\zeta)$ is called an oricycle at $\zeta$, and will be denoted by $K_{r}(\zeta)$, or simply $K(\zeta)$ without specifying $r$. The half of $K_{r}(\zeta)$ lying to the right of the radius at $\zeta$ as viewed from the origin will be denoted by $K_{r}^{+}(\zeta)$ and $K_{r}^{-}(\zeta)$ denotes the left half of $K_{r}(\zeta)$ analogously.

Suppose that $0<r_{1}<r_{2}<1$. Let $r_{3}\left(0<r_{3}<1\right)$ be so large that the circle $|z|=r_{3}$ intersects both of the horocycles $h_{r_{1}}(\zeta)$ and $h_{r_{2}}(\zeta)$. We define the right horocyclic angle $H_{r_{1}, r_{2}, r_{3}}^{+}(\zeta)$ at $\zeta$ with radii $r_{1}, r_{2}, r_{3}$ to be

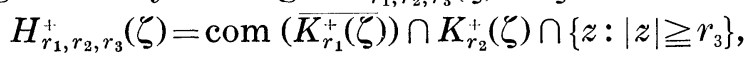

where the bar denotes closure and comp denotes complement, both relative to the plane. The corresponding left horocyclic angle is denoted $H_{r_{1}, r_{2}, r_{3}}(\zeta)$. We write $H_{r_{1}, r_{2}, r_{3}}(\zeta)$ to denote a hyrocyclic angle at $\zeta$ without specifying whether it be right or left, or simply $H(\zeta)$ in the event $r_{1}, r_{2}, r_{3}$ are arbitrary.

We assume the reader to be familiar with the rudiments of the cluster sets.

$C_{V}(f, \zeta)$, the angular cluster set of $f(z)$ at $\zeta$ on a Stolz angle $V(\zeta)$; $C_{K}(f, \zeta)$, the oricyclic cluster set of $f(z)$ at $\zeta$ on an oricycle $K(\zeta)$;

$C_{H}(f, \zeta)$, the horocyclic angular cluster set of $f(z)$ at $\zeta$ on a horocyclic angle $H(\zeta)$.

A point $\zeta \in \Gamma$ is said to be a horocyclic angular Plessner point 
(oricyclic Plessner point) of $f(z)$ provided that

$$
C_{H}^{+}(f, \zeta)=W \text { and } C_{H}^{-}(f, \zeta)=W\left(C_{K}(f, \zeta)=W\right)
$$

for each right and left horocyclic angle (each oricycle) at $\zeta$.

A point $\zeta \in \Gamma$ is called a horocyclic angular Fatou point (oricyclic Fatou point) of $f(z)$ with a horocyclic angular Fatou value (an oricyclic Fatou value) $w \in W$ provided that

$$
C_{H}^{+}(f, \zeta)=\{w\} \text { and } C_{H}^{-}(f, \zeta)=\{w\}\left(C_{K}(f, \zeta)=\{w\}\right)
$$

for each right and left horocyclic angle (each oricycle) at $\zeta$.

Suppose a set $A \subset \Gamma$ and a point $\zeta=e^{i \theta} \in \Gamma$ are given. For a $\varepsilon>0$, we denote an $\operatorname{arc}\left\{e^{i \theta^{\prime}} ; \theta-\varepsilon<\theta^{\prime}<\theta+\varepsilon\right\}$ by $\Gamma(\varepsilon, \zeta)$. Let $\gamma(\zeta, \varepsilon, A)$ be the largest of arcs contained in $\Gamma(\varepsilon, \zeta)$ and not intersecting with $A$. The set $A$ is of porosity of the order $\alpha, 0<\alpha \leqq 1$ (or simply of porosity $(\alpha)$ ) at $\zeta$, if

$$
\varlimsup_{\varepsilon \rightarrow 0} \frac{1}{\varepsilon}\{\gamma(\zeta, \varepsilon, A)\}^{\alpha}>0 .
$$

A is of porosity ( $\alpha$ ) on $\Gamma$ if it is so at each $\zeta \in A$. A set which is a countable sum of sets of porosity $(\alpha)$ is said to be of $\sigma$-porosity $(\alpha)$.

A set of $\sigma$-porosity $(\alpha)$ is of the first Baire category.

It is easily seen that a measurable set which is of porosity (1) on $\Gamma$ has no points of density. Therefore every measurable set of $\sigma$ porosity (1) on $\Gamma$ is of measure 0 . But there exists the set, which is of measure 0 and not of $\sigma$-porosity (1) (see [6], p. 75).

2. A $K H(K V)$-singular point is the point $\zeta \in \Gamma$ such that $C_{K}(f, \zeta)$ $\neq C_{H}(f, \zeta)\left(C_{K}(f, \zeta) \neq C_{V}(f, \zeta)\right)$ for some pair of $K(\zeta)$ and $H(\zeta)(K(\zeta)$ and $V(\zeta))$. The set of all $K H(K V)$-singular points is denoted by $E_{K H}(f)$ $\left(E_{K V}(f)\right)$.

A $K K$-singular point is the point $\zeta \in \Gamma$ such that $C_{K^{\prime}}(f, \zeta) \neq C_{K^{\prime \prime}}(f, \zeta)$ for some pair of oricycles $K^{\prime}(\zeta)$ and $K^{\prime \prime}(\zeta)$. The set of all $K K$-singular points is denoted by $E_{K K}(f)$.

Let $\left\{r_{i}\right\}_{i=1}^{\infty}$ be a sequence of all rational numbers satisfying $0<r_{i}<1$, and let $\left\{D_{n}\right\}$ be a sequence consisting of all closed circles of the plane $W$ having rational radii $r_{n}$ and centers with rational coordinates.

For a $\varepsilon>0$, we set $U_{\varepsilon}(\zeta)=\{z ;|z-\zeta|<\varepsilon\}$. We denote $K_{r_{p}}(\zeta)$ by $\dot{K}_{p}(\zeta)$ and $H_{r_{k}, r_{l}, r_{m}}(\zeta)$ by $H_{k, l, m}(\zeta)$.

Lemma 1. Let $\zeta \in A \subset \Gamma$. Suppose $A$ is not of porosity (1) at a point $\zeta \in A$. Then for fixed $r_{p}, r_{k}, r_{l}, r_{m}, K_{p}(\zeta) \cap U_{\bullet}(\zeta)$ is covered by the set $M=\bigcup_{\xi \in A} H_{k, l, m}(\xi)$ supposed $\varepsilon>0$ is sufficiently small.

Proof. Without loss of generality, we may assume that $\zeta=1$. Now we suppose that there exists a sequence $z_{\nu}=x_{\nu}+i y_{\nu}(\nu=1,2,3, \ldots)$ such that $z_{\nu} \in K_{p}(1) \cap U_{\iota}(1)-M$ and $z_{\nu} \rightarrow 1$. For each $z_{\nu}$, points $R_{1}\left(z_{\nu}\right)$, $S_{1}\left(z_{y}\right), R_{2}\left(z_{\nu}\right), S_{2}\left(z_{\nu}\right)$ on $\Gamma$ are decided as follows.

$R_{1}\left(z_{\nu}\right)\left(S_{1}\left(z_{\nu}\right)\right)$ is the point on $\Gamma$ such that the point $z_{\nu}$ lies on the right half of $h_{r_{l}}\left(R_{1}\left(z_{\nu}\right)\right)\left(h_{r_{k}}\left(S_{1}\left(z_{\nu}\right)\right)\right)$; 
$R_{2}\left(z_{\nu}\right)\left(S_{2}\left(z_{\nu}\right)\right)$ is the point on $\Gamma$ such that the point $z_{\nu}$ lies on the left half of $h_{r_{l}}\left(R_{2}\left(z_{\nu}\right)\right)\left(h_{r_{k}}\left(S_{2}\left(z_{\nu}\right)\right)\right)$.

Let $z_{\nu}=r_{\nu} e^{i \theta_{\nu}}$. We immediately have

$\overline{R_{1}\left(z_{\nu}\right) S_{1}\left(z_{\nu}\right)}$ (the arc length connecting $R_{1}\left(z_{\nu}\right)$ and $S_{1}\left(z_{\nu}\right)$ )

$$
\begin{aligned}
& =R_{2}\left(z_{\nu}\right) S_{2}\left(z_{\nu}\right)=\cos ^{-1}\left\{\frac{2\left(1-r_{l}\right)-\left(1-r_{\nu}^{2}\right)}{2\left(1-r_{l}\right) r_{\nu}}\right\}-\cos ^{-1}\left\{\frac{2\left(1-r_{k}\right)-\left(1-r_{\nu}^{2}\right)}{2\left(1-r_{k}\right) r_{\nu}}\right\}, \\
& \overline{R_{i}\left(z_{\nu}\right) 1}=\theta_{\nu}-(-1)^{i} \cos ^{-1}\left\{\frac{2\left(1-r_{l}\right)-\left(1-r_{\nu}^{2}\right)}{2\left(1-r_{l}\right) r_{\nu}}\right\} \quad(i=1,2) \text {, } \\
& \overline{S_{i}\left(z_{\nu}\right) 1}=\theta_{\nu}-(-1)^{i} \cos ^{-1}\left\{\frac{2\left(1-r_{k}\right)-\left(1-r_{\nu}^{2}\right)}{2\left(1-r_{k}\right) r_{\nu}}\right\} \quad(i=1,2) .
\end{aligned}
$$

Since $z_{\nu} \in K_{p}(1), \quad\left|\theta_{\nu}\right|<\cos ^{-1}\left\{\frac{2\left(1-r_{p}\right)-\left(1-r_{\nu}^{2}\right)}{2\left(1-r_{p}\right) r_{\nu}}\right\}$. We set

$$
\begin{gathered}
\varepsilon_{\nu}=\max \left\{\overline{R_{1}\left(z_{\nu}\right) 1}, \overline{S_{1}\left(z_{\nu}\right) 1}, \overline{R_{2}\left(z_{\nu}\right) 1}, \overline{S_{2}\left(z_{\nu}\right) 1}\right\} . \\
\lim _{\nu \rightarrow \infty} \frac{\overline{R_{1}\left(z_{\nu}\right) S_{1}\left(z_{\nu}\right)}}{\sqrt{1-r_{\nu}}}>0 \text { and } \varepsilon_{\nu}=O\left(\sqrt{1-r_{\nu}}\right) \text { as } \nu \rightarrow \infty .
\end{gathered}
$$

Since $\left\{R_{1}\left(z_{\nu}\right) S_{1}\left(z_{\nu}\right)\right.$ (the arc connecting $R_{1}\left(z_{\nu}\right)$ and $\left.\left.S_{1}\left(z_{\nu}\right)\right) \cup R_{2}\left(z_{\nu}\right) S_{2}\left(z_{\nu}\right)\right\}$ $\cap A=\phi$, we have $\gamma\left(1, \varepsilon_{\nu}, A\right) \geqq \overline{R_{1}\left(z_{\nu}\right) S_{1}\left(z_{\nu}\right)}$.

$$
\lim _{\varepsilon \rightarrow 0} \frac{1}{\varepsilon} \gamma(1, \varepsilon, A) \geqq \lim _{\nu \rightarrow \infty} \frac{1}{\varepsilon_{\nu}} \overline{R_{1}\left(z_{\nu}\right) S_{1}\left(z_{\nu}\right)} \geqq \lim _{\nu \rightarrow \infty} \frac{\sqrt{1-r_{\nu}}}{\varepsilon_{\nu}} \frac{\overline{R_{1}\left(z_{\nu}\right) S_{1}\left(z_{\nu}\right)}}{\sqrt{1-r_{\nu}}}>0,
$$

and obtain a contradiction to the assumption that 1 is not a point of porosity (1) for $A$. Therefore, for $\varepsilon>0$ small enough, $K_{p}(1) \cap U_{\varepsilon}(1)$ is covered by the set $M=\bigcup_{\xi \in A} H_{k, l, m}(\xi)$.

Lemma 2 (Yanagihara [5, Theorem 1]). Let $f: D \rightarrow W$. Then $E_{K K}(f)$ is of the type $G_{\delta \sigma}$ and $\sigma$-porosity (1).

Theorem 1. Let $f: D \rightarrow W$. Then $E_{K H}(f)$ is of the type $G_{\delta \sigma}$ and $\sigma$-porosity (1).

Proof. $E_{n, k, l, m}$ is the set of points $\zeta \in \Gamma$ such that the set

$$
\left\{w=f(z) ; z \in H_{k, l, m}(\zeta)\right\}
$$

lies at a distance $\geqq r_{n}$ from $D_{n}$. $F_{n, p, q}$ is the set of points $\zeta \in \Gamma$ such that the set

$$
\left\{w=f(z) ; z \in K_{p}(\zeta), \frac{1}{3 q}<\operatorname{dis}(z, \zeta)<\frac{1}{q}\right\}
$$

has common points with $D_{n}$.

Then $E_{n, k, l, m}$ is closed and $F_{n, p, q}$ is open. We put

We will show

$$
\boldsymbol{F}_{n, p}=\bigcap_{t=1}^{\infty} \bigcup_{q=t}^{\infty} \boldsymbol{F}_{n, p, q} \text { and } \boldsymbol{A}_{n, k, l, m}, \cap \boldsymbol{F}_{n, p} .
$$

$$
E_{K H}(f)=\left(\bigcup_{n, k, l, m, p} A_{n, k, l, m, p}\right) \cup E_{K K}(f) .
$$

Take a point $\zeta \in E_{K H}(f)$ and $\zeta \notin E_{K K}(f)$. There exist $K(\zeta)$ and $H(\zeta), K(\zeta) \supset H(\zeta)$, for which $C_{K}(f, \zeta) \supsetneq C_{H}(f, \zeta)$. Choose number $p$ and $s$ such that $K_{p}(\zeta) \supset K(\zeta)$ and

$$
D_{s} \cap C_{K}(f, \zeta) \neq \phi, \quad \operatorname{dis}\left(D_{s}, C_{H}(f, \zeta)\right)>5 r_{s} .
$$


Then we can find numbers $k, l, m$ such that $H(\zeta) \supset H_{k, l, m}(\zeta)$ and dis $\left(D_{s}, f(z)\right)>4 r_{s}$ for $z \in H_{k, l, m}(\zeta)$. If $D_{n}$ is a disk with radius $r_{n}=2 r_{s}$ and concentric with $D_{s}$, dis $\left(D_{n}, f(z)\right)>r_{n}$ for $z \in H_{k, l, m}(\zeta)$, which shows $\zeta \in E_{n, k, l, m}$. In view of (5) there exists an infinite number of $q$ such that

$$
D_{n} \cap\left\{w=f(z) ; z \in K_{p}(\zeta), \frac{1}{3 q}<\operatorname{dis}(z, \zeta)<\frac{1}{q}\right\} \neq \phi,
$$

which shows $\zeta \in F_{n, p}$. Thus $\zeta \in A_{n, k, l, m, p}$ and

$$
E_{K H}(\zeta) \subset\left(\bigcup_{n, k, l, m, p} A_{n, k, l, m, p}\right) \cup E_{K K}(f) .
$$

Take a point $\zeta \in\left(\bigcup_{n, k, l, m, p} A_{n, k, l, m, p}\right) \cup E_{K K}(f) . \quad$ If $\zeta \in E_{K K}(f)$, clearly we have $\zeta \in E_{K H}(f)$. If $\zeta \in \bigcup_{n, k, l, m, p} A_{n, k, l, m, p}, C_{H_{k, l}, m}(f, \zeta) \cap D_{n}=\phi$ from (1) and $C_{K_{p}}(f, \zeta) \cap D_{n} \neq \phi$ from (2). Thus we have $C_{H_{k}, l, m}(f, \zeta) \neq C_{K_{p}}(f, \zeta)$ and $\zeta \in E_{K H}(f)$. Hence $\left(\bigcup_{n, k, l, m, p} A_{n, k, l, m, p}\right) \cup E_{K K}(f) \subset E_{K H}(f)$.

Since $E_{K K}(f)$ is of type $G_{\delta \sigma}$ by Lemma 2, the equality (4) shows that $E_{K H}(f)$ is type $G_{\delta \sigma}$.

According to Lemma $1, E_{K K}(f)$ is of $\sigma$-porosity (1), so that it remains to prove that $A=A_{n, k, l, m, p}$ is of porosity (1).

Suppose $A$ is not of porosity (1) at a point $\zeta \in A$. Then for sufficiently small $\varepsilon>0, K_{p}(\zeta) \cap U_{\varepsilon}(\zeta)$ is covered by the set $\bigcup_{\xi \in A} H_{k, l, m}(\xi)$ by Lemma 1. Thus if $z \in K_{p}(\zeta) \cap U_{\sigma}(\zeta)$, there is a point $\xi \in A=A_{n, k, l, m, p}$, $z \in H_{k, l, m}(\xi)$. Therefore $w=f(z)$ lies at a distance $\geqq r_{n}$ from $D_{n}$, and $C_{K_{p}}(f, \zeta) \cap D_{n}=\phi$. This contradicts with $\zeta \in F_{n, p}$. Thus the porosity (1) of $A$ is proved.

Theorem 2 (Yanagihara [5, Theorem 2]). Let $f: D \rightarrow W$. Then $E_{K V}(f)$ is of the type $G_{\delta \sigma}$ and of $\sigma$-porosity $(1 / 2)$.

3. Now we can state some precisions and generalizations of the results of Bagemihl [1, Theorem 1, Theorem 2, Theorem 4 and Remark 3] and Dragosh [3, Theorem 1, Remark 2 and Corollary 2].

Theorem 3. Let $f: D \rightarrow W$. Then a horocyclic angular Fatou point of $f(z)$ is an angular Fatou point of $f(z)$ except on a set of $\sigma$ porosity (1).

Proof. According to Theorem 1, except on a set of $\sigma$-porosity (1), a horocyclic angular Fatou point of $f$ is an oricyclic Fatou point of $f$, which is an angular Fatou point of $f$ by the fact $C_{K}(f, \zeta) \supset C_{V}(f, \zeta)$.

Theorem 4. Let $f: D \rightarrow W$. Then an angular Fatou point of $f(z)$ is a horocyclic angular Fatou point except on a set of $\sigma$-porosity $(1 / 2)$.

Proof. This is an analogous deduction from Theorem 2.

Theorem 5. Let $f: D \rightarrow W$. Then an angular Plessner point of $f(z)$ is a horocyclic angular Plessner point of $f(z)$ except on a set of $\sigma$-porosity (1).

Proof. By the fact $C_{K}(f, \zeta) \supset C_{V}(f, \zeta)$, an angular Plessner point 
of $f$ is an oricyclic Plessner point of $f$, which is a horocyclic angular Plessner point of $f$ except on a set of $\sigma$-porosity (1) according to Theorem 1.

Theorem 6. Let $f: D \rightarrow W$. Then a horocyclic angular Plessner point of $f(z)$ is an angular Plessner point of $f(z)$ except on a set of $\sigma$ porosity $(1 / 2)$.

Proof. This is an analogous deduction from Theorem 2.

4. We have not yet established the complete structual characterizations of set $E_{i}(i=1,2,3,4)$ such that (a horocyclic angular Fatou point is an angular Fatou point on $E_{1}$, an angular Fatou point is a horocyclic angular Fatou point on $E_{2}$, an angular Plessner point is a horocyclic angular Plessner point on $E_{3}$. a horocyclic angular Plessner point is an angular Plessner point on $E_{4}$, for some functions.

But we establish only one special result in this direction here

In proving next Theorem 7 , we use the function $f(z)$ constructed by Yanagihara [5].

Lemma 3. Let $E \subset \Gamma$ be a closed everywhere disconnected set. Then there exists a bounded holomorphic function $f(z)$ with the following properties:

1) At every $\zeta \in \operatorname{comp}(F), f(z)$ is continuous. Therefore, $\zeta$ is both an angular Fatou point and a horocyclic angular Fatou point.

2) Each point $\zeta \in F$ is an angular Fatou point having an angular limit of modulus 1.

3) Each point $\zeta \in F$ at which the set $F$ is of porosity (1/2) is not a horocyclic angular Fatou point.

Proof. Comp $(F)$ consists of a countable number of ares $\left(\zeta_{\nu}^{\prime}, \zeta_{\nu}^{\prime \prime}\right)$. For a constant $r(0<r<1), h_{r}\left(\zeta_{\nu}^{\prime}\right) \cap h_{r}\left(\zeta_{\nu}^{\prime \prime}\right) \neq \phi$ except at most finite number of $\nu$ 's. Let $z_{\nu, 1}^{\prime}=z_{\nu, 1}^{\prime \prime}$ be the one of intersection points of $h_{r}\left(\zeta_{\nu}^{\prime}\right)$ and $h_{r}\left(\zeta_{\nu}^{\prime \prime}\right)$ which is nearer to $\Gamma$. For each exceptional index $\nu$, let $z_{\nu, 1}^{\prime}$ be the left one of intersection points of $h_{r}\left(\zeta_{\nu}^{\prime}\right)$ and $|z|=1-r$, and let $z_{\nu, 1}^{\prime \prime}$ be the right one of intersection points of $h_{r}\left(\zeta_{\nu}^{\prime \prime}\right)$ and $|z|=1-r$. Next, let $z_{\nu, n}^{\prime}$ be the point on $h_{r}\left(\zeta_{\nu}^{\prime}\right)$ such that

$$
\frac{1-\left|z_{\nu, n}^{\prime}\right|}{\left|\zeta_{\nu}^{\prime}-z_{\nu, n}^{\prime}\right|}=\frac{1}{2} \frac{1-\left|z_{\nu, n-1}^{\prime}\right|}{\left|\zeta_{\nu}^{\prime}-z_{\nu, n-1}^{\prime}\right|} \quad(n=2,3,4, \cdots)
$$

The sequence $\left\{z_{\nu, n}^{\prime \prime}\right\}$ on $h_{r}\left(\zeta_{\nu}^{\prime \prime}\right)$ is defined analogously.

Then the Blaschke product

$$
f(z)=\prod \frac{\overline{z_{\nu, n}^{\prime}}}{\mid \overline{z_{\nu, n}^{\prime} \mid}} \frac{z-z_{\nu, n}^{\prime}}{1-\overline{z_{\nu, n}^{\prime}}} \prod \frac{\overline{z_{\nu, n}^{\prime \prime}}}{\mid{z_{\nu, n}^{\prime \prime} \mid}_{1-}^{\prime \prime}} \frac{z-z_{\nu, n}^{\prime \prime}}{1-\overline{z_{\nu, n}^{\prime \prime}} z}
$$

has the properties asserted in Lemma 3.

Now, we shall prove here the property 3) only.

If we choose appropriate constants $r_{1}, r_{2}, r_{3}$ and $\zeta \in F$ is a point at 
which the set $F$ is of porosity $(1 / 2), H_{r_{1}, r_{2}, r_{3}}(\zeta)$ contains an infinite number of points from $\left\{z_{\nu, n}^{\prime}, z_{\nu, n}^{\prime \prime}\right\}$. Hence, for each point $\zeta$ at which the set $F$ is of porosity $(1 / 2)$, if $\zeta$ is a horocyclic Fatou point of $f(z)$, then the horocyclic Fatou value at $\zeta$ must be 0 , so that by the theorem of Lindelöf, the angular Fatou value at $\zeta$ must be 0 , too. But this contradicts the property 2 ).

Theorem 7. For each set of $\sigma$-porosity $(1 / 2) E \subset \Gamma$, there exists a bounded analytic function $f(z)$ for which each $\zeta \in E$ is an angular Fatou point and is not a horocyclic angular Fatou point.

Proof. $E$ can be represented in the form of a countable sum of sets $E_{n}$ nowhere dense on $\Gamma: E=\cup E_{n}$. Suppose that a set $E^{\prime}$ is a countable sum of closed everywhere disconnected sets $\overline{E_{n}}: E^{\prime}=U \overline{E_{n}}$. Then $E^{\prime}$ can also be represented in the form of a not more than countable sum of closed sets $F_{k}$ without common points (Dolzehnko [4], English translation, p. 8).

From this construction, it is evident that each point $\zeta \in \overline{E_{n}}$ at which some $E_{n}$ is of porosity $(1 / 2)$ is also a point of porosity $(1 / 2)$ for some $F_{k}$.

Now, for each $\boldsymbol{F}_{k}$ we construct a sequence of zeros $\left\{z_{\nu, n}^{k}\right\}$ and Blsschke product $f_{k}(z)$, as in Lemma 3 . Set

$$
f(z)=\sum 2^{-k} f_{k}(z) \text {. }
$$

If $\zeta \in E_{k}$, all $f_{\nu}(z)(\nu \neq k)$ are continuous at $\zeta$ (Lemma 3,1$\left.)\right)$, and $f_{k}(z)$ has an angular limit of modulus 1 (Lemma 3, 2)). Hence each point $\zeta \in E$ is an angular Fatou point. On the other hand, if $\zeta \in E_{n}$ is a point of porosity $(1 / 2)$ for $F_{k}$, then $\zeta$ is not a horocyclic angular Fatou point of $f_{k}(z)$ (Lemma 3,3$)$ ). Thus each point $\zeta \in E$ is not a horocyclic angular Fatou point of $f(z)$.

Theorem 7 corresponds to Theorem 4 . In this connection, it is natural to ask the following question: Is it true that there fold analogous results to Theorem 7 corresponding to other theorems in section 3 ? I would guess that it is positive even for holomorphic functions.

\section{References}

[1] Bagemihl, F.: Horocyclic boundary properties of meromorphic functions. Ann. Acad. Sci. Fenn., A I, 385, 1-18 (1966).

[2] Dragosh, S.: Horocyclic boundary behavior of meromorphic functions. J. d'Anal. Math., 22, 37-48 (1969).

[ 3 ] - : Horocyclic cluster sets of functions defined in the unit disk. Nagoya Math. J., 35, 53-82 (1969).

[4] Dolzhenko, E. P.: Boundary properties of arbitrary functions. Izvestija, Acad. Nauk SSSR, 31, 3-14 (1967).

English translation: Math. of the USSR-IZVESTIJA, 1, 1-12 (1967).

[5] Yanagihara, N.: Angular cluster sets and oricyclic cluster sets. Proc. Japan Acad., 45, 423-428 (1969).

[6] Collingwood, E. F., and Lohwater, A. J.: The Theory of Cluster Sets. Camb. Univ. Press (1966). 